\title{
Knowledge, Attitude and Practice towards Dengue Fever among University Students
}

\section{Fatimah S, RN, MSc ${ }^{1,3}$, Wan Nur Asikin WO, RN ${ }^{1}$, Nor Azean M, RN ${ }^{1}$, Muhammad Amin AZ, RN², MSc, Razif D, MSc ${ }^{1,3}$}

${ }^{1}$ Faculty of Health Sciences, UniversitiTeknologi MARA, Selangor Branch, PuncakAlam Campus, 42300 PuncakAlam, Selangor, Malaysia

${ }^{2}$ Faculty of Health Sciences, UniversitiTeknologi MARA, Pulau Pinang Branch, Bertam Campus, 12300 Kepala Batas, Pulau Pinang, Malaysia

${ }^{3}$ Maternofetal and Embryology Research Group, Faculty of Medicine, Sungai Buloh Campus, UniversitiTeknologi MARA (UiTM)

\section{Abstract}

Background: Dengue is a viral disease transmitted to humans by the bite of infested females of the main vector Aedes aegypti and to lesser extent Aedes albopictus mosquitoes. Knowledge regarding dengue fever (DF) is very important among students to create awareness for practicing the prevention and increase the student's attitude

Corresponding Author: Muhammad Amin AZ aminzaki@uitm.edu.my

Received: 22 September 2019 Accepted: 4 October 2019 Published: 10 October 2019

Publishing services provided by Knowledge E

(c) Muhammad Amin AZ et al. This article is distributed under the terms of the which permits unrestricted use and redistribution provided that the original author and source are credited.

Selection and Peer-review under the responsibility of the ICHT 2019 Conference Committee.
S OPEN ACCESS levels. Objective: To assess the knowledge, attitude and practice regarding Dengue Fever among University Students. Methods: The study design was cross-sectional design. This study was conducted in at nine campuses in two states in Malaysia. A purposive sampling technique was used of 1144 students who residing in this public university collegesfrom the selected setting and willing to participate in the study. Self-administered questionnaire consisted of four sections, socio-demographic profile, the knowledge, attitude and practice related to dengue/ mosquito control. The data were entered and analyzed using IBM Statistical Packages for Social Science (SPSS) for Window, version 23 and descriptive analyses were executed for each variable. Results: In total, $74 \%$ of the participants were female and $26 \%$ male, ages ranged from 18 to 39 years. Overall $97.8 \%$ of them ever heard of DF and the predominant source of the information was media (86.4\%). Fever, joint pains and headache was well known as a symptom of DF for most of students. They knew that Aedes mosquitoes transmit DF (94.8\%) and stagnant water is the main source for mosquito breeding (94.8\%). Many participants aware that removal of stagnant water (89.2\%), using mosquito repellents (88.9\%), tightly covering water containers (86\%) and the use of windows screen and bed nets (82.5\%) able to prevent mosquito breeding and reduced contact with mosquitoes. Consulting a physician for DF (92.7\%), taking plenty of rest (86.1\%) and drink plenty of water (81.4\%) was the best management for DF. Most of the students agreed $(97.3 \%)$ that dengue is a serious illness. They agreed that communities should actively participate in controlling the vectors of dengue virus (94.7\%) in which controlling the breeding places of mosquitoes, a good strategy to prevent DF (97.5\%). Thus, this study revealed that the respondents had perceived inadequate knowledge $(85.0 \%)$, poor attitude (58.0\%) and poor practice (88.0\%) of Dengue fever. Conclusion: In conclusion, although this study discovered that the students' had basic knowledge, aware of the seriousness of the disease and practiced some level of prevention related to DF but the needs of educational programs adapting transformational learning and strategies 
by the university such as developing student's friendly and continuous information regarding DF as part of health promotion strategies and the needs of holistic approach, community participation and cooperation is important to impart knowledge and foster good practices in the prevention and controlling of dengue not only in the campus but trigger good practice among the community in general.

Keywords: Knowledge, Attitude \& Practice, Dengue Fever, University Students.

\section{Introduction}

Dengue is a viral disease transmitted to humans by the bite of infested females of the main vector Aedes aegypti and to lesser extent Aedes albopictus mosquitoes. World Health Organization (WHO) has classified dengue into three categories according to disease severity like Dengue Fever (DF), Dengue Hemorrhagic Fever (DHF), and Dengue Shock Syndrome (DSS) [1]. Dengue fever is caused by dengue viruses (DENV) which is the most common mosquito borne viral disease in the world [2].

WHO classified Dengue as the most important mosquito-borne viral disease in the world. Over 2.5 billion people over $40 \%$ of the world's population are now at risk from dengue. DF is most common in Southeast Asia and the western Pacific islands, but the disease has been rapidly spreading in Latin America and the Caribbean. As stated [3], WHO and Centers for Disease Control and Prevention recommended the extensive community educational campaigns that emphasize reducing vector breeding sites as an effective way of dengue prevention. For now, the best prevention is to reduce mosquito habitat in areas where dengue fever is common.

In Malaysia, dengue is perceived as a highly contagious health threat with escalating trend of infection. The average number of dengue cases and death tolls had recorded a surge of $14 \%$ and $8 \%$, respectively per annum, over the years of 2000-2010 [4]. In the first half of the year of 2015, a total number of 59866 dengue cases and 165 deaths had been reported in Malaysia [4].

According to statistic provided by Health Unit UiTM Cawangan Selangor, which are in PuncakAlam, PuncakPerdana, Selayang, Sungai Buloh and Dengkil, on 2012 until 2017 there were 46 new cases confirmed dengue infected. In 2012 there was 3 cases of Dengue fever, 4 cases in 2013, 5 cases in 2014, 6 cases in 2015, 10 cases in 2016 and 2017 was 18 cases of Dengue fever have been reported. Health Centre of UiTM Shah Alam reported 332 cases confirmed dengue infected with no deaths since 2014 until 
2017. In 2014, 49 cases were reported by Health Officer, 55 cases in 2015, 102 cases of Dengue Fever and 1 cases of Dengue Hemorrhagic Fever (DHF) in 2016 and in 2017, 126 cases of Dengue fever were reported.

The number of dengue cases in UiTMCawanganNegeri Sembilan was 49 confirmed dengue infected cases with no death reported since 2014 until 2017 by Health Unit, which are in Seremban, Kuala Pilah and Rembau campuses. In 2014, 7 cases of Dengue fever, whereas in 2015, 8 cases were reported. The dengue cases were increasing to 10 cases in 2016 with 1 cases of DHF and 23 cases in 2017.

This increasing incidence of dengue fever and its more severe forms clearly call attention to the importance of health behaviors and attitudes the prevention of dengue fever, which may be enhanced by studies addressing the knowledge, attitude and practice related to dengue fever. In order to educate public to do so, knowledge about dengue infection need to be delivering. By doing this study among students in UiTM Shah Alam, Selangor and Negeri Sembilan, researcher hope to assess their level of knowledge, attitude and practice towards dengue infection. The data obtained later is hope to be guideline in upcoming prevention step that need to be carry out to these students in order to prevent dengue spreading.Thus, this study aimed to identify knowledge, attitude and practice regarding Dengue Fever among University Students.

\section{Methods}

The researcher has chosen a cross-sectional study design in this study since this research attempt to observe or assess level of knowledge, attitude and practice of the respondents related to Dengue Fever. The study has been conducted in UiTM from two state; Selangor and Negeri Sembilan.

The students from six campuses in Selangor; UiTM Shah Alam, PuncakAlam, PuncakPerdana, Sungai Buloh, Selayang and Dengkil and three campuses in Negeri Sembilan; UiTMRembau, Seremban 3 and Kuala Pilah, were involved in this study.UiTM Shah Alam was the highest rate of dengue cases which is 126 cases in 2018. UiTM in Selangor was second Campus among all UiTM in Malaysia that involves in high rate of dengue cases, 23 cases in 2018 was recorded whereas in UiTM in Negeri Sembilan there were 18 cases in 2018 . 


\subsection{Sample size}

Using convenient sampling technique, this study was conducted among 1144 students who residing in this public university colleges over a period of four months from December 2018 to April 2019.The sample size was determined according to the setting in ensuring the result will be able to portray the outcome of the study. The sample size calculated by using Raosoft Sample Size Calculation with 95\% confidence level and 5\% of margin error. Table 1 showed the inclusion and exclusion criteria of the respondent.

TABLE 1: Respondent's criteria.

Inclusion Criteria
i. All undergraduate students who residing in
UiTM Colleges in these two states; Selangor
and Negeri Sembilan.
ii. They were able to understand English and
willing to participate in this study.

Inclusion Criteria

i. All undergraduate students who residing in and Negeri Sembilan.

willing to participate in this study.

\section{Exclusion Criteria}

i. The exclusion criteria were students who in their practical session will be taken into this consideration for this study.

\subsection{Instruments}

The instrument used in this study was a self-administrated questionnaire adapted from $[3,5]$ with the aim is to obtain the knowledge, attitude and practice relating to Dengue Fever among the UiTM students.

Self-administered questionnaire consisted of 67 questions divided in four sections, socio-demographic profile, the knowledge, attitude and practice related to dengue/ mosquito control.This questionnaire was divided into four sections; Section A consists of demographic data, Section B is to assess the knowledge, Section C is about attitude and Section $D$ is about practice toward prevention dengue fever. It was an open ended questionnaire.

\subsubsection{Section A: Socio-demographic Data of the respondents and the information regarding Dengue Fever}

There were 8 questions in Section A which comprise of demographic data such as the respondent's age, gender, faculty, level of education, and year of study, heard about dengue fever, sources of information and experience of Dengue Fever. 


\subsubsection{Section B: Knowledge}

Questionnaire in Section B consists of 39 items of "YES", "NO" and "DON'T KNOW" questions about symptoms, transmission and management regarding Dengue Fever. For each knowledge item, a correct was coded '1' and wrong answer was coded ' 0 '. Therefore, the total knowledge scores ranged from 0 to 39. The student level of knowledge was measured using McDonald's standard of learning outcome as stated [6]. The level of knowledge have been categorized into; very low $(<60 \%)$, low $(60-69)$, moderate (70-79), high (80-89), and very high (90-100). Thus, knowledge score of $\geq 80 \%$ is consider in adequate.

\subsubsection{Section C: Attitude}

Section $C$ questions used to determine the attitude regarding Dengue Fever among the UiTM students. It consists of 9 items rated on a 5-point Likert scale that was intended to identify. The response choices for items in the Likert scales of part $\mathrm{C}$ were "strongly disagree", "disagree", "not agree" "nor disagree", "agree" and "strongly agree". For attitude items, a positive attitude was coded ' 1 ' and negative was coded ' 0 '. Thus, the total score ranges from 0-9. A score of 8 and above is considered good attitude.

\subsubsection{Section D: Practice}

Section D consists of 11 questions to identify the practice regarding Dengue Fever. The response choices for items in the true false questions of part D were yes, no and don't know. Practice items was coded ' 1 ' for 'yes' and ' 0 ' was for 'no' while 'don't know' also was coded with ' 0 '. The total scores ranged from 0 to 11. A score of 9 and above is considered good practice.

\subsection{Reliability}

The reliability of the questionnaire was tested through IBM SPSS version 21 by analyzing the Cronbach's Alpha value of the questionnaire. The data used to test the reliability of the questionnaire was taken from the pilot study that was through before the data collection for the actual study. The value of the Cronbach's Alpha after analyzing the pilot study data was 0.84 , which specify this is a good questionnaire. 


\subsection{Data collection procedure}

The study was commenced following approval from the Institutional Research Ethic Committee and for ethical considerations, consent was obtained from the studied campuses and individual participants, and the confidentiality of information was assured.The respondents have been chosen based on inclusion criteria. The data collection was conducted using multi-choice questionnaire. The data collection was obtained from students around residential colleges, libraries and at the student activity centres. Selfintroduction and brief explanation about the study was given to respondents before conducting the data collection. Respondents were informed that all the information was kept confidential by the researcher and we're not being made public unless disclosure is required by law.

\subsection{Data analysis}

The data were entered and analyzed using IBM Statistical Packages for Social Science (SPSS) for Window, version 23 and descriptive analyses were executed for each variable.

\section{Results}

\subsection{Demography}

In total, 1144 students participated in the study. Of these participants, $74 \%$ were female and $26 \%$ male. Ages ranged from 18 to 39 years. Half of the participants study under the cluster of Science \& Technology (50.1\%), 38.4\% from Business \& Management while 11.5\% were from Social Science \& Humanities. Overall $97.8 \%$ of them ever heard of Dengue Fever and the predominant source of the information was media (86.4\%). Table 2 showed the level of education and year of study of respondents.

\subsection{Knowledge, Attitude and Practice}

Majority of students were able to correctly identify typical symptoms of dengue such as fever (89.1\%), joint pains (87.1\%), headache (77.4\%,) and nausea/vomiting (76.2\%). While approximately half of the participants incorrectly identified pain behind the eyes (47.5\%), abdominal pain (50.3\%) and diarrhea is a common in dengue fever (45.3\%). 
TABLE 2: Level of education and Year of study.

\begin{tabular}{|l|c|c|}
\hline Level of education & $\mathbf{n}$ & $\%$ \\
\hline Diploma & 349 & 30.5 \\
\hline Degree & 694 & 60.7 \\
\hline Foundation & 101 & 8.8 \\
\hline Year of study & $\mathbf{n}$ & $\%$ \\
\hline $1^{s t}$ & 362 & 31.6 \\
\hline $2^{\text {nd }}$ & 426 & 37.2 \\
\hline $3^{\text {rd }}$ & 249 & 21.8 \\
\hline $4^{\text {th }}$ & 107 & 9.4 \\
\hline
\end{tabular}

They knew that Aedes mosquitoes transmit Dengue fever (94.8\%) and stagnant water is the main source for mosquito breeding (94.8\%). However, almost half of them answered incorrectly for the mosquitoes bite time; day time only (40.1\%) and night time only (49.1\%).

Many participants aware that removal of stagnant water (89.2\%), using mosquito repellents $(88.9 \%)$, tightly covering water containers $(86 \%)$ and the use of windows screen and bed nets (82.5\%) able to prevent mosquito breeding and reduced contact with mosquitoes.

Consulting a physician for dengue fever (92.7\%), taking plenty of rest (86.1\%) and drink plenty of water (81.4\%) was the best management for Dengue fever. However, $65 \%$ of the participants misunderstand that paracetamol is a suitable drug for dengue treatment.

Most of the students agreed (97.3\%) that dengue is a serious illness. They agreed that communities should actively participate in controlling the vectors of dengue virus (94.7\%) in which controlling the breeding places of mosquitoes, a good strategy to prevent DF (97.5\%).

They practiced covering water jars at hostel's room (75\%), draining water from flower pot (70.6\%) and always ensured that the water tanks at hostel's toilet covered (69.2\%).However only $24.8 \%$ of the participants usedtemephos, eg: Abate ${ }^{\circledR}$ to eliminate of mosquito larvae, 31.8\% examine mosquito larvae both indoors and outdoors, $35.8 \%$ regularly inspect their hostel's area and $36.3 \%$ participated in community cleaning activities.

Thus, this study showed that University Student perceived inadequate knowledge (85.0\%), poor attitude (58.0\%) and poor practice (88.0\%) toward Dengue fever. Table 3 showed the level of KAP on dengue fever among university student. 
TABLE 3: Level of KAPon Dengue Fever among University Student.

\begin{tabular}{|c|c|c|}
\hline Result of KAP & $\mathbf{n}$ & $\%$ \\
\hline \multicolumn{3}{|l|}{ Knowledge } \\
\hline Adequate & 177 & $15.0 \%$ \\
\hline Inadequate & 967 & $85.0 \%$ \\
\hline \multicolumn{3}{|l|}{ Attitude } \\
\hline Good & 484 & $42.0 \%$ \\
\hline Poor & 694 & $58.0 \%$ \\
\hline \multicolumn{3}{|l|}{ Practice } \\
\hline Good & 132 & $12.0 \%$ \\
\hline Poor & 1012 & $88.0 \%$ \\
\hline
\end{tabular}

\section{Discussion}

This study was carried out to assess the knowledge, attitudes and practice related to dengue fever among university students.

Fever, joint pains and headache was well known as a symptom of dengue fever for most of students and these similar with other studies [3, 5, 7-9]. They also showed a good knowledge in managing DF as most of them knew to consult a physician, taking plenty of rest and drink plenty of water. These congruent with previous study [5].

However, these study showed deficiencies knowledge related to DF as half of the participants unable to answer correctly that pain behind the eyes, abdominal pain and diarrhea as a symptom of dengue fever. And this was similar with previous studies [5, 9]. The need of early detection of DF will determine the effective management.

These situation quite worrying as lots of the participants agreed that paracetamol is a suitable drug to treat dengue fever and these similar with studies $[3,5,8,10]$.

Regarding preventive practices in our study, most of the participants aware that removal of stagnant water, using mosquito repellents, tightly covering water containers and use windows screen and bed nets in order to prevent mosquito breeding and reduced contact with mosquitoes. These similar with other studies [5].

Our study has demonstrated the deficiency of knowledge regarding attributes of dengue infection especially the Aedes mosquito bite time as most of them thought that dengue mosquitoes bite in day or at night time only. These similar with other studies $[5,7-9]$. This is important to aware of specific bite times as part of preventive measure.

This study reported that most of the participants know that Aedes mosquitoes transmit dengue fever and stagnant water is the main source for mosquito breeding. These similar $[3,5,8]$. In line with this, most of them practice covering water jars at hostel's 
room, draining water from flower pot and ensured that the water tanks at hostel's toilet covered and these similar with studies [5].

They agreed that dengue is a serious illness $[3,10]$ in which actively participation by thecommunities needed in controlling the breeding places of mosquitoes as a good strategy to prevent DF. These similar with studies [5, 10].

However, their attitudes are not in line with their practices as most of them showed poor practice in participating in community cleaning activities. In addition, the poor practice in using of temephos, eg: Abate $^{\circledR}$ to eliminate of mosquito larvae, examine mosquito larvae both indoors and outdoors and inspect hostel's area regularly. These similar with studies $[3,10]$.

These indicate the need of behavioural change in transforming action to improve preventive measures.

As cases of dengue fever are still on the rise. It is important to initiate transformational learning in order to gain better knowledge and best practice in controlling of dengue fever among university students. This strategy can be effective when performed regularly, especially when our university students are mobilized and educated about vector control.

\section{Conclusion}

In conclusion, although this study discovered that the students' had basic knowledge, aware of the seriousness of the disease and practiced some level of prevention related to dengue fever but the needs of educational programs adaptingtransformational learning and strategies by the university such as developing student's friendly and continuous information regarding DF as part of health promotion strategies and the needs of holistic approach, community participation and cooperation is important to impart knowledge and foster good practices in the prevention and controlling of dengue not only in the campus but trigger good practice among the community in general.Further research need to find association between demographic and knowledge, attitude and practice toward dengue fever.

\section{Declaration}

This research was carried out in accordance with the regulations of UniversitiTeknologi MARA (UiTM), Malaysia. Thank for UiTM Research Ethics Committee, Faculty of Health Sciences upon approval of this research with reference no. 600-IRMI (5/1/6). 


\section{Acknowledgments}

Authors would like to acknowledge the UniversitiTeknologi MARA for supporting this study. We would also like to acknowledge all the professionals, health personnel, and all the respondents participated in this study.

\section{Conflict of Interest}

The authors have no conflict of interest to declare.

\section{References}

[1] Binsaeed, A., Sahli, A., Noureldin, E., Mohammed, W., Dafalla, \& Dahlan, A., (2015). Knowledge, Attitudes and Preventive Practices of Dengue Fever among Secondary School Students in Jazan, Saudi Arabia. Current World Environment, 10(3), 747-757.

[2] World Health Organization (WHO), (2009). Preventive and control of dengue hemmorhagic fever. Dengue Guidelines for Diagnosis, Treatment, Prevention and Control, 160. Retrieved from https://doi.org/10.1590/S0074-02761992000700024.

[3] Yussof,F., M., Hassan, A.,Zin,T.,Hussin, T., M., A., R., Kadarman, N., \&Umar. R., (2017). Knowledge Of Dengue Among Students In Universiti Sultan Zainal Abidin (UNISZA), Terengganu, Malaysia And The Influence Of Knowledge Of Dengue On Attitude And Practice. Journal of Fundamental and Applied Sciences. 9(2), 199-216.

[4] Kamel, M., N., A., M., Gnanakkan, B., D., Selvarajah, F., Z., F., M., I., H., Geetha Selvarajah, Jabar, S. A., \& Hamid, S., A. (2017). The KAP Study on Dengue among Community in Taman Salak Baiduri. International Journal of Science and Healthcare Research, 2(9), 19-25.

[5] Payghan, B., S., \& Chandram, S. (2014). Knowledge Attitude And Practices Regarding Dengue Infection Among Pre-University College Students. International Journal of Medical Science and Clinical Inventions, 1(7), 371-378.

[6] Jager, A. de. (2002). An integrated and holistic approach to assessment in outcomebased learning in South Africa. South African Education, 1, 1-16.

[7] Abbasi, A., Abbas, K., Arooj, S., Habib, N., Aziz, W., and Ashaq, A. (2016). Dengue Fever: A Statistical Analysis Regarding Awareness about Dengue among University Students in Azad Kashmi. Journal of HealthcareCommunications. 2472-1654

[8] Rafaqat Botaa, Mushtaq Ahmeda, Muhammad Salah Jamali,\& Adnan Aziz (2014). Knowledge, attitude and perception regarding dengue fever among university 
students of interior Sindh. Journal of Infection and Public Health. 7,218-223.

[9] Ujala Nayyar, Umar Farooq Dar, Muhammad Zahid Latif, RaziHaider, Tahir Mahmud \&RahilaNizami (2013). Knowledge, Awareness and Practices about Dengue Fever among University Students. Pakistan Journal of Medical and Health Sciences.

[10] Amanah, M., A., Abdullah, H., \& Ghafar, N., A. (2018). Knowledge attitude and practice on dengue among university students. International Journal of Community Medicine and Public Health, 5(11), 4720-4724. 